LAWRENCE LIVERMORE N A T IO N A L LABORATORY

\title{
3D Computations and Experiments
}

Richard Couch, Doug Faux, Dana Goto, Dan Nikkel

April 6, 2004 
This document was prepared as an account of work sponsored by an agency of the United States Government. Neither the United States Government nor the University of California nor any of their employees, makes any warranty, express or implied, or assumes any legal liability or responsibility for the accuracy, completeness, or usefulness of any information, apparatus, product, or process disclosed, or represents that its use would not infringe privately owned rights. Reference herein to any specific commercial product, process, or service by trade name, trademark, manufacturer, or otherwise, does not necessarily constitute or imply its endorsement, recommendation, or favoring by the United States Government or the University of California. The views and opinions of authors expressed herein do not necessarily state or reflect those of the United States Government or the University of California, and shall not be used for advertising or product endorsement purposes.

This work was performed under the auspices of the U.S. Department of Energy by University of California, Lawrence Livermore National Laboratory under Contract W-7405-Eng-48. 


\section{D Computations and Experiments}

Performing organization Lawrence Livermore National Laboratory
Livermore, CA 94551

Principal

investigator

Co-Investigators

\author{
Richard Couch \\ (925) 422-1655 \\ Doug Faux \\ (925) 423-9705 \\ Dana Goto \\ (925) 422-3804 \\ Dan Nikkel \\ (925) 422-6415
}

DoD contacts

DoD contacts
Bob Garrett

NSWC, Dahlgren

Scott Schoenfeld

ARL

Andrew Geltmacher

NRL name

affiliation

Project funding

\begin{tabular}{|c|c|c|c|c|c|c|}
\hline FY & $\mathbf{0 4}$ & $\mathbf{0 5}$ & $\mathbf{0 6}$ & $\mathbf{0 7}$ & $\mathbf{0 8}$ & $\mathbf{0 9}$ \\
\hline $\mathbf{\$ K}$ & 1600 & 1600 & 1600 & 1600 & 1600 & 1600 \\
\hline
\end{tabular}

This project consists of two activities. Task A, Simulations and Measurements, combines all the material model development and associated numerical work with the materialsoriented experimental activities. The goal of this effort is to provide an improved understanding of dynamic material properties and to provide accurate numerical representations of those properties for use in analysis codes. Task B, ALE3D Development, involves general development activities in the ALE3D code with the focus of improving simulation capabilities for problems of mutual interest to DoD and DOE. Emphasis is on problems involving multi-phase flow, blast loading of structures and system safety/vulnerability studies. 


\section{Task A: Simulations and Measurements (1000K)}

\section{Technical Problem}

The understanding of material properties and the ability to produce accurate representations of those properties in numerical simulations of dynamic events is of great importance to the development and fielding of weapon systems. Examples where material properties play a critical role include explosively formed penetrators, long rod penetrators, shaped charges, penetration mechanics, fracture and fragmentation. The physical phenomena that determine material properties and material response to loading and deformation can be quite complex. Often a physical understanding of macroscopic response can only be understood in terms of models for the microscopic properties and response.

The goal of this project is to develop physically based models of the 3D response of materials to dynamic loading that can be utilized in simulation codes to address the applications listed above. The focus will be on attaining a predictive capability for the evolution of constitutive response and for the initiation and evolution of fracture. The project will combine experimentation and model development activities. The experiments will be designed to evaluate microscopic properties and response in order to provide the data and knowledge required to construct macroscopic models for use in continuum level simulation codes.

\section{Expected Payoffs}

The understanding and potential control of material properties that can arise from the multiscale model approach allows one to envisage significant advances in the technology associated with the manufacture of metal components. Many characteristics of metal parts are directly related to attributes that can only be understood by characterizing the properties of the utilized material at the meso- and micro-scales. If one could translate such knowledge into models that would both represent and evolve those properties through a forming process, then one would have attained a greater degree of control of the attributes that determine the performance of the formed part in its intended application. The manufacture of liners and the dynamic formation of shaped charge jets and explosively formed projectiles are the most ready examples of relevant applications. The same physical considerations can be applied to the potential for understanding and control of fracture and fragmentation.

\section{Technical Status and Issues}

A combined experimental and computational approach is required to produce models with predictive capability. The model development activity has focused on the representation of anisotropic material response, improved representations of constitutive properties at high strain rates, and improved damage and failure models. The 
experimental work will focus on providing the data required to elucidate the basic physical mechanisms and to validate the derived models.

The multiscale modeling approach has demonstrated considerable promise for the development of models of material response. Information obtained from experimental and theoretical studies at the lower length scales is contributing to the formulation and utility of continuum level models. Continued research is required to bring these models to a state at which they can regularly contribute to design and development activities.

\section{Summary of Current Year's Work}

\section{Model Development}

Activities in this area include developing models to represent anisotropic material properties, an improved representation for constitutive properties at high strain rates, and improved material damage and failure models. This activity is part of a broader effort intending to incorporate the results of investigations at the atomic-, micro-, and mesoscale levels into continuum models for the above processes. This effort also supports continued development of a variety of macro-scale models for the ALE3D user community.

Progress has been made in the development of a formalism for treating anisotropic plasticity. The implementation in ALE3D has been extended to include dynamic evolution of the yield surface and rate dependent hardening. The goal is to associate models and model parameters with specific microstructural characteristics in order to capture the physics of material deformation. The physical models for determining the course of this evolution in yield surface are still under development. Some success has been attained in trying to predict the rotation observed in some shaped charge jets and attributed to anisotropic liner properties. The attempt has been made to start with measured microstructural features of a fabricated liner, produce an anisotropic response model from the observed properties, and then predict the observed rotation in a 3D calculation of the liner collapse and jet formation. Qualitative success has been attained in that jet rotation is observed. Continued work is needed to get better quantitative agreement with the amount of rotation.

An effort has been undertaken to develop improved strength models for plastic bonded high explosives (PBXs). Such materials generally exhibit extreme time and temperature dependent mechanical response that is dominated by the binder material. Experimental data has suggested that the visco-plastic mechanisms may effect the response as much as visco-elasticity, even at small strains. A basic visco-plastic model has been developed for LX-17 or PBXs, and a framework for the thermal effects has been formulated. We are in the process of doing the implementation of this visco-plastic model into ALE3D, with model evaluations to follow.

Crystal plasticity modeling has been extended to allow for more accurate treatment of strong shocks. The goal of this meso-scale modeling is to shed light on the physical 
processes by which micro-structural features affect macro-response. Application areas include yield surface evolution under deformation, strain localization, and free surface effects.

Simulations of dynamic fracture experiments have produced results that indicate that a model representing the actual statistical distribution of damage initiation sites and their effect on local fracture response can be used to predict fragment characteristics such as fragment size distributions. This modeling activity suggested a series of experiments to test this hypothesis. This will be discussed further below.

\section{Experiments}

Recent activity has focused on developing a novel experimental test technique to investigate dynamic deformation and fracture under moderate stress-triaxiality loading conditions. The experimental technique is developed in collaboration with Dennis Grady, and as such is referred to as the Grady Gas Gun eXperiment (G3X); see Figure 1. Initial G3X results appeared to yield promising data for biaxially deformed thin plates. Using a recently acquired 3D image correlation photogrammetry package, which measures real-time specimen displacement in three-dimensions, deformation of the bulged, flat plate was determined as a function of position. Figure 2 shows an example of the as-bulged disk and illustrates the deformation is symmetric about the center/apex of the bulge and produces a deformation pattern (z-displacement) in the form of a 'bulls eye' pattern, in which the deformation level is symmetric and decreases with increasing distance from the center of the disk. Upon closer inspection of this bulged disk, a small crack was observed on the face in contact with the copper buffer; see Figure 3. Upon subsequently cross-sectional metallographic inspection, the subsurface material clearly revealed internal cavitation in the form of a crack oriented parallel to the disk surfaces, reminiscent of a "spall" plane; see Figure 4. Based on computer code design calculations this observation was unexpected (and not entirely desirable). As a result of the observation of internal cavitation of the G3X sample, the test is currently be redesigned and is not a part of near-term planned work under this MOU effort. 


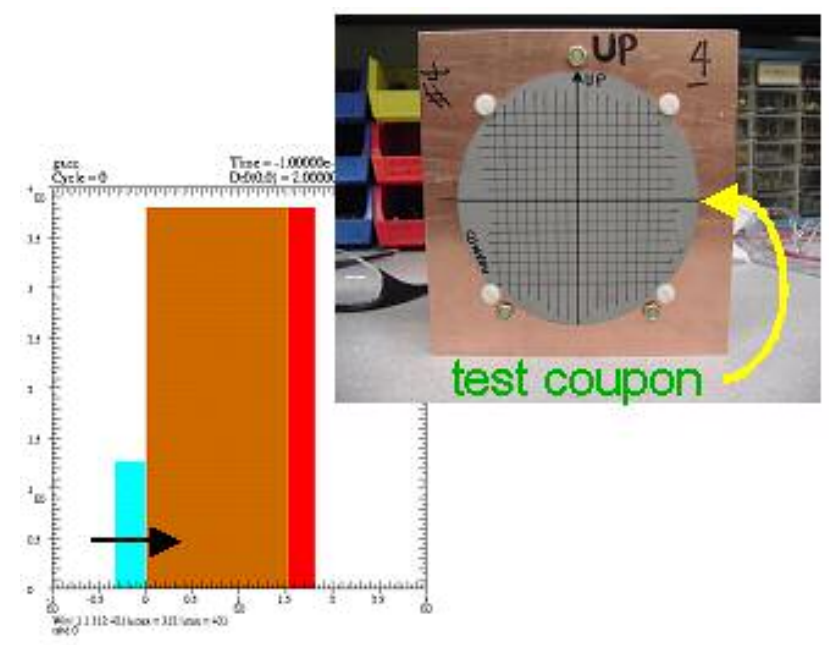

Figure 1. Grady gas gun test set-up. Brown (in the computational model) represents the copper buffer, and red represents the steel target. Aqua represent the copper flyer plate.
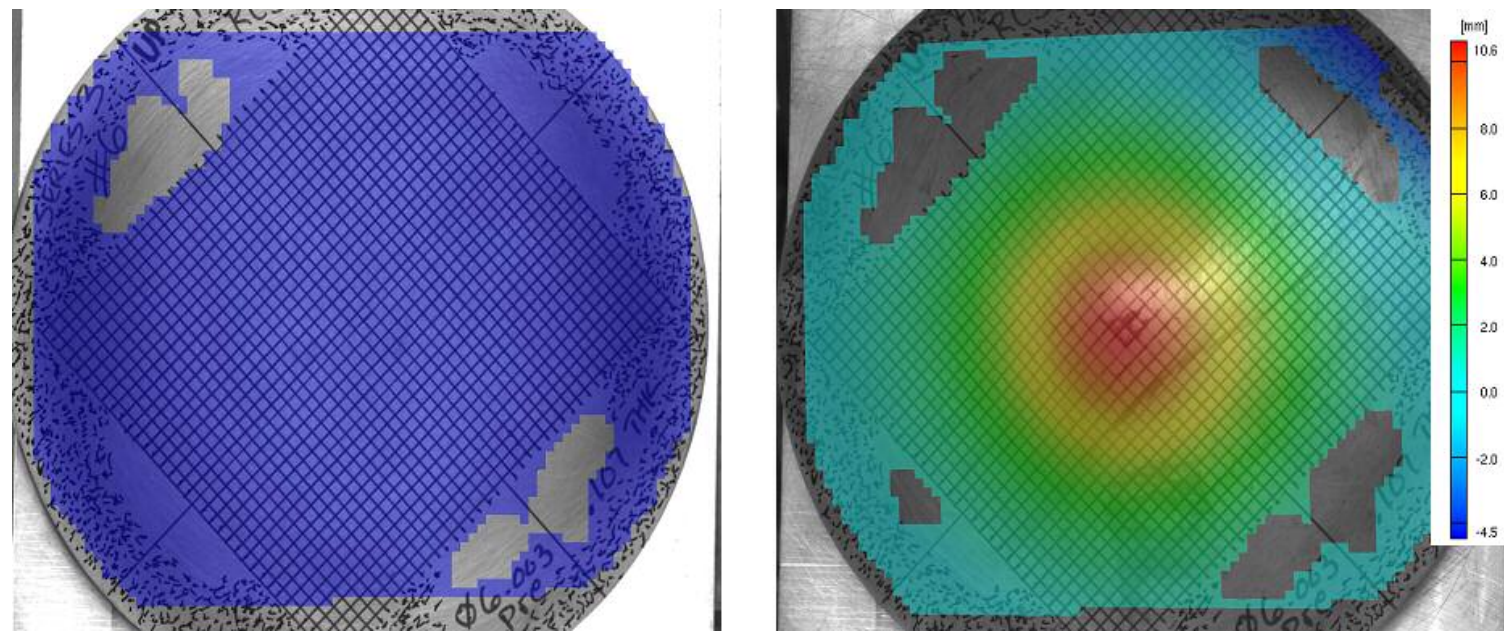

Figure 2. Macro photo of pre-test and post-test, bulged sample overlayed with a pseudocolor contour of z-displacement computed from the 3D image correlation photogrammetry. 


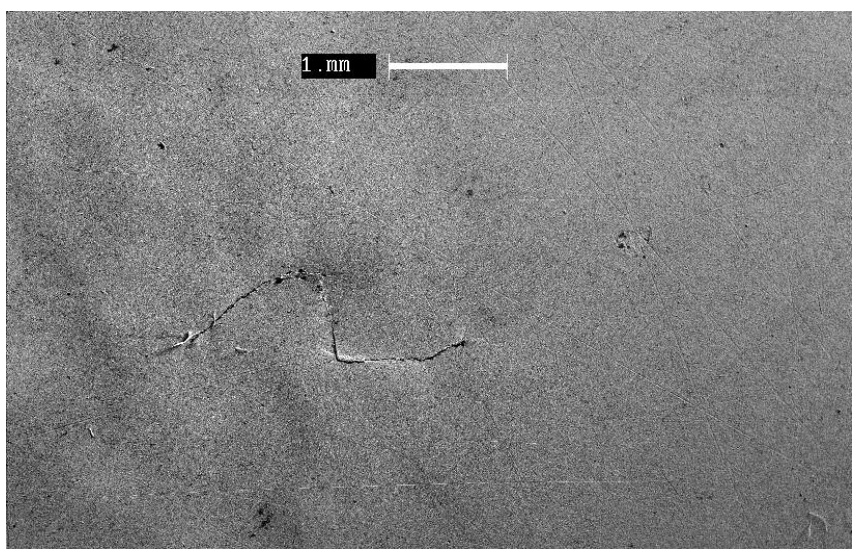

Figure 3. Fine scale crack on the AerMet-100 target plate in the vicinity of the bulge apex. This face of the target plate was originally in contact with the copper buffer during the test.

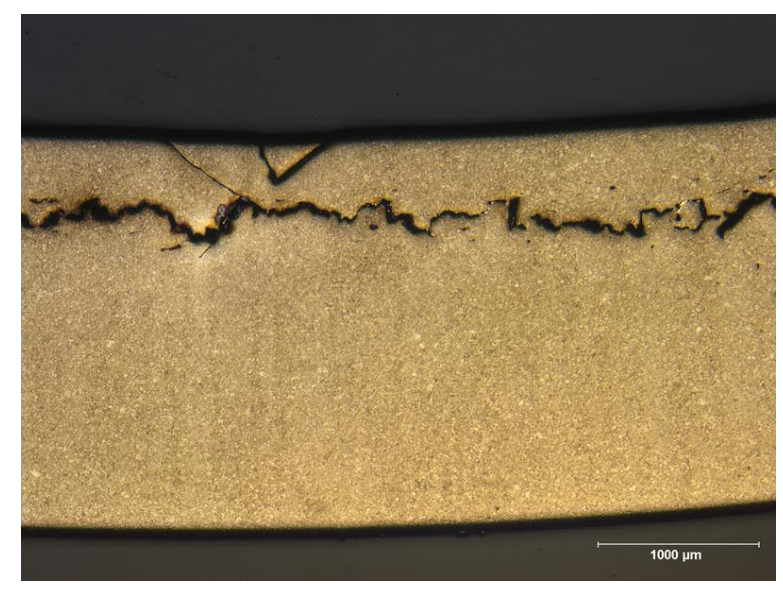

Figure 4. Optical micrography showing internal cavitation of the AerMet-100 following a G3X test. The metallograph was taken orthogonal to the fine scale crack, shown in Figure 2.

Near term efforts will focus on a more fundamental materials modeling study. This new activity is intended to examine and better understand length scale issues in ductile fracture using sub-continuum scale experiments. A more detailed discussion will be given in the 'Planned Work' section.

Continued work has been performed toward characterization and testing the polycrystal virtual test sample. The initial orientation imaging microscopy (OIM) was completed of the Tantalum two-dimensional virtual test sample (VTS-2D). This first procedure characterizes the initial material condition prior to deformation. Currently the sample is being prepared for the first tensile strain increment (roughly 0.02 strain). 
The VTS-2D testing provides important validation data for on-going crystal plasticity modeling. Currently, based on the testing schedule, the VTS-2D test is anticipated as being completed by end of FY04. As such, this experimental task will be phased into the newer sub-continuum scale experiments. In addition to studying length scale issue in ductile fracture, these newer lower length-scale tests will study material localization issues from a meso-scale level.

\section{Planned Work}

\section{Model Development}

Code development activities will continue to focus on providing enhanced capabilities in the areas of advanced material models. Activities in this area include developing models to represent anisotropic material properties, an improved representation for constitutive properties at high strain rates, and improved material damage and failure models. This activity is part of a broader effort intending to incorporate the results of investigations at the atomic-, micro-, and meso-scale levels into continuum models for the above processes. Particular emphasis will be placed on modeling the various case fracture and fragmentation experimental studies underway. Physically based material failure models must be tied to subscale phenomena to be generally useful and predictive. The model development will utilize the data being made available by the experimental activities and will suggest the most useful experiments for its purposes.

\section{Experiments}

\section{Polycrystal Virtual Test Sample}

The current plan is to complete work on the polycrystal virtual test sample. Currently the sample is being prepared for the first tensile strain increment (roughly 0.02 strain). Prior to testing the sample will be 'speckled' to prepare for real-time deformation measurements. The speckled sample will employ a recently purchased diagnostic system, which allows full-field optical displacement and strain measurements to be made using white light speckle image correlation. In short, three-dimension deformation information can be measured during testing, in real-time. Following this first deformation increment, the sample will be re-OIM'ed to determine grain orientations. This procedure will be continued until the sample has accumulated approximately $0.20-$ 0.25 strain.

\section{Fracture Experiments}

Developing physically based models and modeling capability of the dynamic fracture process necessitates an understanding of the relationship between microstructure and fracture response. The planned work intends to address this capability through laboratory experiments to evaluate the statistics of fracture, under well-controlled boundary and initial conditions, and to understand the nature of these statistics within the context of the 
material microstructure. New insight into correlating properties to microstructure is a critical component of the multiscale modeling approach to fracture mechanics.

The scope of this study involves a novel and unique approach entailing detailed quantitative metallography, determination of a material Representative Volume Element (RVE), and micro-scale mechanical testing; see Figure 5. Distributions of microstructural features, such as size, morphology and spatial, and degree of clustering, etc. of one or more second phase particle populations will be measured. Such metrics of the microstructure can be applied to describing a characteristic length scale as well as to creating or synthesizing a virtual, notional microstructure. This notional microstructure is hereafter referred to as the Representative Volume Element (RVE); see Figure 6.

An objective of this study is to study the physics of deformation of the RVE, viz., the roles of the matrix and particle populations in the deformation leading up to fracture, and motivate a RVE-based fracture model. To support RVE model development, micro-scale mechanical testing will be conducted. The purpose of the testing is to quantify and understand the nature of the fracture statistics of the bulk material (macro-tests), RVE (micro-tests) and sub-scale RVE (micro-tests). Measuring directly either the bulk properties or that of the RVE will produce statistics for a fracture model, but not provide the insight as to explicit roles of the matrix and particle populations in the fracture process. Sub-scale RVE testing (and modeling) is anticipated as allowing more direct probing, by both measurements and observations, of the influence of microstructure components, their individual and collective contributions, on mechanical response.
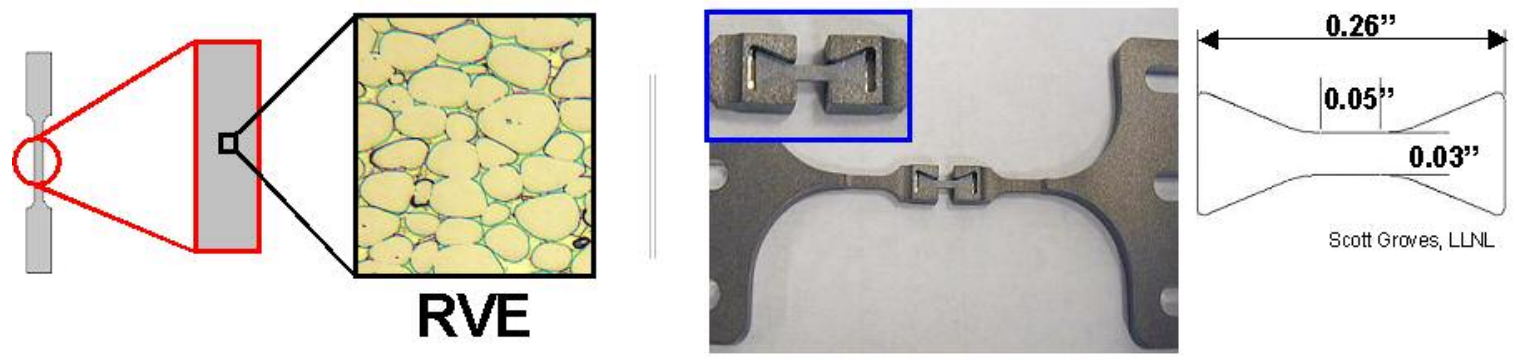

Figure 5. Schematic description of the RVE and the novel LLNL micro-tensile test. The $\mathrm{RVE}$ (e.g., W-Ni Fe alloy is shown) is presumed as the minimum representative material volume that represents the continuum material microstructure and properties. Microtensile tests are intended to test the RVE and material volumes smaller than the RVE to measure continuum and sub-continuum size scale material response. 


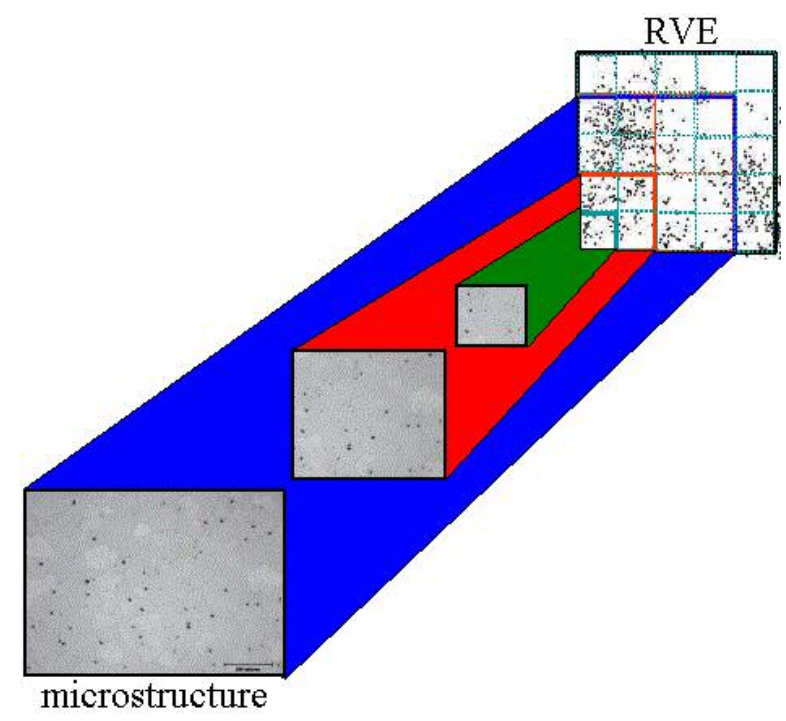

Figure 6. Graphical representation between microstructure and RVE. Based on the microstructure, a material RVE may encompass a small volume of material (defined by the green box) or a larger volume, as defined by the blue box. Based on the experimental determination of the RVE (defined by the length scale of microstructural constituents) a notional RVE can be synthesized for computational modeling.

In conjunction with these experiments, numerical computations will be performed to support experiment development and interpretation and analysis of experimentally measured data. The quantitative metallography data and mechanical test data will be applied toward the development of an RVE-base, statistics-motivated, fracture model applicable to dynamic fracture.

Initially, model materials are selected to facilitate developing the framework for conducting these sub-size scale analyses, i.e., material microstructures that can readily be metallurgically characterized. Such materials include low carbon steels that have been heat-treated to produce a range of microstructures from as quenched to spheroidized microstructures. Initial interest is in 1045 steel as a material to use to validate the approach. Targeted materials might include the steels 43xx, 41xx and AerMet 100. Tungsten heavy alloys are also being considered as potential candidates for applying this investigation methodology.

\section{Technology Transition}

The modeling and experimental activities are communicated to DoD parties through the TCG process, by individual technical interchanges and by publication in the open literature. The material models being developed are generally resident in ALE3D, which is available to DoD facilities and contractors. These models are usually published in the open literature and can be made available as programmed routines for implementation in other simulation codes. 


\section{Milestones}

\begin{tabular}{|c|c|c|c|c|c|}
\hline Tasks & FY05 & FY06 & FY07 & FY08 & FY09 \\
\hline \multicolumn{6}{|l|}{ Materials Characterization } \\
\hline \multicolumn{6}{|l|}{ Pre-test quantitative metallography } \\
\hline \multicolumn{6}{|l|}{ Post-test quantitative metallography } \\
\hline \multicolumn{6}{|l|}{ Test Design } \\
\hline \multicolumn{6}{|l|}{ RVE sample design } \\
\hline \multicolumn{6}{|l|}{ Sub-scale RVE sample design } \\
\hline \multicolumn{6}{|l|}{ Mechanical Testing } \\
\hline Bulk material & & & & & \\
\hline RVE-scale & & & & & \\
\hline Sub-scale RVE & & & & & \\
\hline Data Analysis (Model Development) & & & & & \\
\hline
\end{tabular}




\section{Task B: ALE3D Development (600K)}

\section{Technical Problem}

Accurate and efficient numerical simulations of hydrodynamic events are of great importance in munitions design and effectiveness activities. Computer simulations allow for rapid and detailed evaluation of warhead concepts at small incremental cost. As resource intensive experiments become less available, the cost effectiveness of numerical modeling will continue to increase.

Traditional hydrocodes are generally pure-Eulerian or pure-Lagrangian in format. The latter provide good geometric resolution, but their utility is severely diminished when large material distortions are encountered. Pure-Eulerian codes are capable of dealing with arbitrarily large material distortions, but satisfactory resolution often generates excessive memory requirements and calculation times. The arbitrary-Lagrange-Eulerian (ALE) format combines the resolution of a Lagrangian code with the capability of an Eulerian code to handle large distortions. LLNL has developed ALE techniques that provide a more efficient use of the computational mesh resulting in shorter calculations that require less computer memory.

ALE3D had its genesis with DYNA3D. The code has been completely rewritten and many of the algorithms have been altered or replaced, but the basic, finite element data structure has been maintained. The code currently treats only hexahedral solid elements, but the connectivity is allowed to be arbitrary in the sense that a single node can be shared with an arbitrary number of elements. This feature in itself leads to significant advantages in zoning efficiency over a regularly connected code like CALE. The grid can also be organized into independently zoned blocks that interact through slide surfaces. Intersecting slide surfaces are allowed as are the opening and closing of voids between blocks. A single-sided slide surface treatment allows the boundary of a block to fold back on itself. Calculations in Lagrangian regions can be performed with beam and shell elements. This capability, coupled with the ability to treat multifluid dynamics with an ALE mesh makes ALE3D a powerful tool.

ALE3D also provides a high level integration of a number of modeling options. Beam and shell elements are available to provide a more powerful structural representation. A version with heat conduction and thermal-chemistry has been developed. An implicit time integration scheme for the solution of the dynamics is also available for some applications. This software provides a unique aggregate of capabilities.

\section{Expected Payoffs}

The implementation of the ALE capability in ALE3D provides distinct advantages to the defense community. The improved resolution available: 
1. provides greater accuracy,

2. allows more detailed investigation of physical phenomena,

3. extends simulation capability to more classes of problems.

Shortened computation times and diminished memory requirements:

1. improve cost effectiveness in R\&D activities,

2. allow numerical modeling capability to be more widely available.

The combined thermal-chemical-mechanical simulation capability allows:

1. treatment of more complex simulations than previously possible,

2. treatment of vulnerability and lethality issue at closer to a full system level.

The availability of structural elements within the 3D ALE code represents a dramatic improvement in capability and efficiency, and will be of direct assistance in a number of applications of importance to the defense community. The availability of fully coupled thermal-mechanical solutions with chemical reaction allows a number of new applications to be addressed in the areas of weapon system vulnerability and lethality.

\section{Technical Status and Issues}

Code development activities are currently focused on providing enhanced capabilities in the areas of

- multiphase flow

- blast loading of structures

- $\quad$ system safety/vulnerability

There is growing emphasis on understanding the response of energetic materials to dynamic stimuli within the context of TCG-1. It is expected that this emphasis will focus more of our efforts under this project in the area of validation of this class of models within ALE3D and disseminating them for use by DoD personnel.

\section{Summary of Current Year's Work}

\section{Multiphase Flow}

Activities in this area have focused on developing a model that will allow the simulation of heavily particle loaded explosive mixtures. Significant interest has developed in 
modeling the collateral effects produced by metal-loaded mixtures in which the metal load contributes significantly to the mass and volume of the explosive. These types of simulations will require new models for treating these complex mixtures. Independent thermodynamic and hydrodynamic states are assigned for each fluid material or phase in order to represent the discrete nature of the mixture. Distinct density, volume fraction, and velocities are assigned to each material. We are currently developing an implementation that will cast the model into a formulation that is consistent with the current ALE techniques used in ALE3D. The model and its implementation have progressed to the stage where validation calculations are being run for realistic geometries. After verification of results against experimental data has occurred the code can be made available for analyses. This is expected to occur late in FY04.

\section{Modeling of Structural Loading}

Several significant developments have occurred relevant to this class of problems. The suite of models for geological materials available in the GEODYN code are available now in ALE3D. These models will be of great value in a variety of weapons effects and penetration mechanics simulations.

An effort to develop and implement more efficient and more flexible representations for rebarred concrete is underway. One can represent the rebar explicitly in a highly resolved representation of the material and obtain excellent results. However, this approach is extremely time consuming in terms of both problem setup and execution. We are investigating approaches that distribute the effects of the rebar. Two methods have been used previously by other researchers with some success. They involve a smeared representation of the rebar through a solid element, and a representation in terms of shell elements with multiple through-thickness points of integration. We will implement both approaches and make modifications as required to attain the accuracy required.

The implicit dynamics model has been used to generate gravity load stresses on a complex structure. The ALE3D results were nearly identical to a loading calculation on the same structure using NIKE3D. This is a significant accomplishment on the path to attaining a completely self-contained structural loading capability within ALE3D.

\section{Distribution and User Interaction}

We have made several visits at DoD facilities to discuss code applications and have had several visits to LLNL by DoD personnel for hands-on code training. Interest in this later activity is growing. A session was held in March for $15 \mathrm{DoD}$ personnel from NAWC, NSWCDD and Eglin AFB. 


\section{Planned Work}

Code development activities will continue to focus on providing enhanced capabilities in the areas of weapon system safety in abnormal environments, multiphase flow, and blast loading of structures. Activity directed towards producing models associated with soft target damage enhancement modeling will be a primary focus area during this period. This activity is well aligned with the multiphase flow component of this project. Additional interactions with the DoD community will be aggressively pursued.

The increased emphasis on "insensitive munitions" within the DoD could impact the focus and scope of the effort. This could translate to greater emphasis on development and validation of models for simulating the response of configurations containing energetic materials to various assaults. This is also a topic of great interest to the DOE community. This project will respond in an appropriate manner.

Several DoD facilities have expressed interest in a capability to couple electrical and magnetic effects to ALE3D. This capability would be of interest to LLNL for internal usage. There is an internal research proposal circulating within LLNL to work on that coupling. Should that proposal be funded we would expect to participate in making that capability available to the DoD community. The tasking of this project would be to facilitate the code and capability transfer to the DoD through code validation exercises and training activities.

Another area in which there is growing DoD interest is in the understanding of structural loading incurred in high $\mathrm{G}$ environments such as material penetration events. This is an area in which LLNL has some experience and an area in which ALE3D has been used. Again, we would expect to participate by facilitating the code and capability transfer to the DoD through code validation exercises and training activities.

\section{Technology Transition}

ALE3D has been provided to over 30 facilities. Most copies have gone to Service Laboratories and Defense contractors, the rest to commercial industries or universities. Code distribution in the past year includes the organizations in the table below. Provided below is the list of organizations that have requested and received copies of the ALE3D code in the past 18 months.

\section{Organization}

Eglin/AFRL/MNAC

Picatinny Arsenal

\section{Contact}

Douglas Nance

Vladimir Gold 
USA Space and Missile Defense Command

Naval Explosive Ordnance Tech Division

NSWC Dahlgren

Naval Research Laboratory

Foils Engineering

ANALYTIX Corp.

Computer Aided Engineering Associates

Alcoa

NAWC, China Lake

USACE ERDL

Research Triangle Institute

Penn. State Univ.

ARL

ACTA
John Tipton

Richard Gold

Robert Nance

Andrew Geltmacher

Marshall Eck

Heros Noravian

Steven Hale

Joe Fridy

Shane Schumacher

James O'Daniel

Robb Laney

Damian Tatum

John Starkenberg

Ron Lambert

\section{Relationship to Ongoing Work in DOE/Services/OSD}

A 3D ALE code with some of the features of ALE3D is under development at SNL.

\section{Benefits to DOE/DoD}

A combination of economic and political factors has placed an increased emphasis on modeling and simulation in DOE and DoD design and evaluation activities. The 3D code development activity supported here is focussed on support of those activities, particularly in the areas of multi-disciplinary and full-system simulations. 


\section{Milestones}

\section{Task B: ALE3D Development}

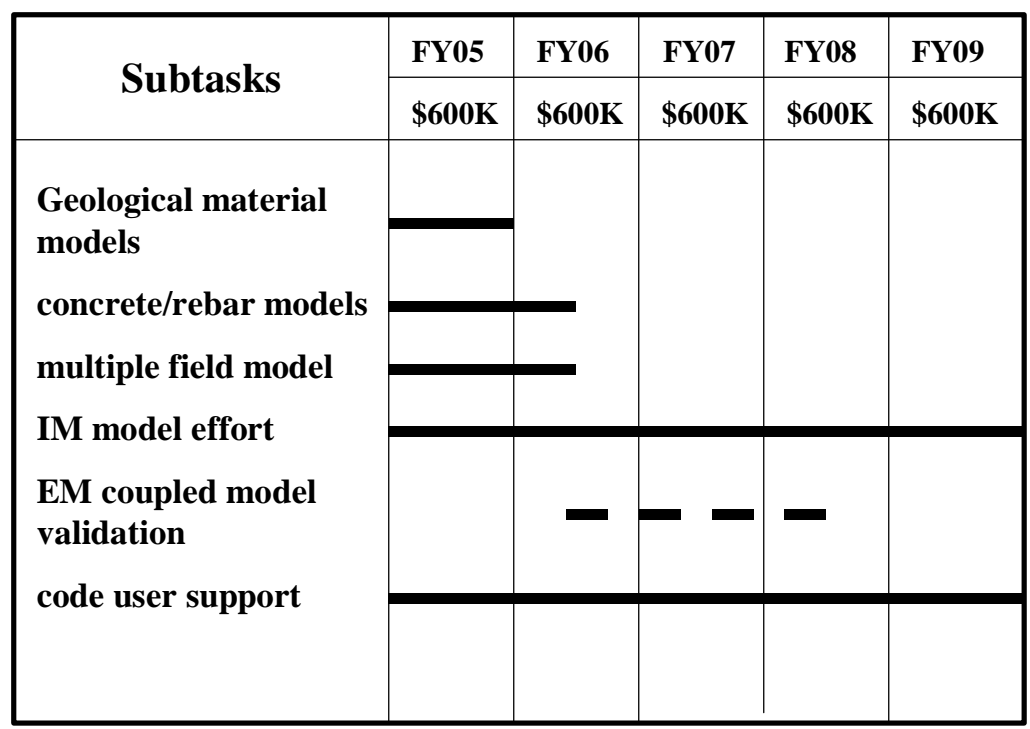

This work was performed under the auspices of the U. S. Department of Energy by University of California, Lawrence Livermore national Laboratory under Contract W7405-Eng-48. 